\title{
Hydrodynamic Modes for Granular Gases
}

\author{
James W. Dufty \\ Department of Physics, University of Florida, Gainesville, Florida 32611 \\ J. Javier Brey \\ Física Teórica, Universidad de Sevilla, E-41080 Sevilla, Spain
}

(Dated: October 31, 2018)

\begin{abstract}
The eigenfunctions and eigenvalues of the linearized Boltzmann equation for inelastic hard spheres $(d=3)$ or disks $(d=2)$ corresponding to $d+2$ hydrodynamic modes, are calculated in the long wavelength limit for a granular gas. The transport coefficients are identified and found to agree with those from the Chapman-Enskog solution. The dominance of hydrodynamic modes at long times and long wavelengths is studied via an exactly solvable kinetic model. A collisional continuum is bounded away from the hydrodynamic spectrum, assuring a hydrodynamic description at long times. The bound is closely related to the power law decay of the velocity distribution in the reference homogeneous cooling state.
\end{abstract}

PACS numbers: PACS numbers: 45.70.-n, 05.20.Dd, 51.10.+y 
The simplest model for a granular gas at low density is given by the Boltzmann equation for smooth, inelastic hard spheres or disks [1]. It provides an appropriate context in which to address a number of fundamental issues. Primary among these is the existence of a macroscopic fluid dynamics analogous to the Navier-Stokes description for real gases. The derivation of hydrodynamic equations from the inelastic Boltzmann equation and identification of expressions for the transport coefficients has been a problem of interest for two decades [2, 3, 4, 5 ].

Accurate prediction of the transport coefficients as a function of the restitution coefficient 6] , and confirmation via Monte Carlo simulation 7], has been accomplished only within the last few years. However, the method used in these derivations (Chapman-Enskog) is formal and has been susceptible to debate. It is of interest therefore to look for a hydrodynamic description in the simplest context of linear response to a small perturbation of the "universal" homogeneous cooling state (HCS). This response is provided by the linearized Boltzmann equation, and the existence of hydrodynamics depends on the spectrum of the operator. Another issue is the isolation of the hydrodynamic spectrum from the remaining "microscopic" excitations, such that the hydrodynamic modes dominate on the long space and time scales. For elastic collisions, this has been proven for "hard" potentials (including hard spheres) 8].

Here a calculation of the hydrodynamic spectrum from the linearized inelastic Boltzmann equation is reported. The transport coefficients are identified and shown to agree with those from the Chapman-Enskog method. Moreover, some support for the isolation of the hydrodynamic spectrum is provided by an exact analysis of a model Boltzmann equation [6, 9]. The model exhibits the dominant features of granular gases, including a non-trivial HCS with algebraic decay at large velocities, discussed extensively for related Maxwell models 10]. It is shown that the pure point spectrum for elastic collisions develops a continuum for any degree of inelasticity, extending toward the hydrodynamic spectrum. However, it remains bounded away from the latter by an amount that is controlled by the power law of the decay of the velocity distribution. This relationship of the spectrum to the reference HCS is unexpected, but it is argued that it is more general than the limitations of the model.

The inelastic nonlinear Boltzmann equation for the density $f(\mathbf{r}, \mathbf{v}, t)$ of particles of mass $m$ at position $\mathbf{r}$ with velocity $\mathbf{v}$ at time $t$ has the form

$$
\left(\partial_{t}+\mathbf{v} \cdot \nabla\right) f=J[f, f]
$$


where $J[f, f]$ is a bilinear functional of $f[1]$. There is no stationary solution to this equation for an isolated system. However, a homogeneous cooling solution (HCS) is assumed to exist with a scaling property similar to the Maxwellian,

$$
f_{H C S}(\mathbf{v}, t)=n_{H} v_{0}^{-d}(t) \chi_{0}(\mathbf{c}), \quad \mathbf{c}=\frac{\mathbf{v}}{v_{0}(t)}
$$

Here $n_{H}$ is the homogeneous density and $v_{0}(t)=\sqrt{2 T(t) / m}$ is the thermal velocity defined in terms of the temperature $T(t)$. The latter obeys the equation $T^{-1} \partial_{t} T=-\zeta_{0}$, where the cooling rate $\zeta_{0}=\zeta\left[\chi_{0}, \chi_{0}\right]$ also is a specified bilinear functional. Both $\chi_{0}$ and $\zeta_{0}$ must be determined self-consistently from the Boltzmann equation

$$
\frac{1}{2} \zeta_{0} \frac{\partial}{\partial \mathbf{c}} \cdot\left(\mathbf{c} f_{H C S}\right)=J\left[f_{H C S}, f_{H C S}\right]
$$

The existence of $f_{H}$ is supported by approximate polynomial expansions [11] and by Monte Carlo simulation [12].

To study the relaxation of small spatial perturbations of the HCS, $\Delta$ is defined by $f=f_{H C S}[1+\Delta]$, and terms up through linear order in $\Delta$ are retained in the Boltzmann equation. The resulting equation is then written in dimensionless form with the velocities scaled relative to $v_{0}(t)$ and the time expressed in terms of $s=\int^{t} d t^{\prime} \nu_{0}\left(t^{\prime}\right)$, where $\nu_{0}(t)$ is the average collision frequency, proportional to $\sqrt{T(t)}$. It is sufficient to consider a single Fourier component $\tilde{\Delta}(\mathbf{k})$ that obeys

$$
\begin{gathered}
\left(\partial_{s}-i \mathbf{k} \cdot \mathbf{c}+\mathcal{L}\right) \tilde{\Delta}=0 \\
\mathcal{L} \tilde{\Delta}=- \\
-\chi_{0}^{-1}\left\{J\left[\chi_{0}, \chi_{0} \tilde{\Delta}\right]+J\left[\chi_{0} \tilde{\Delta}, \chi_{0}\right]\right. \\
\left.-\frac{\zeta_{0}}{2} \frac{\partial}{\partial \mathbf{c}} \cdot\left(\mathbf{c} \chi_{0} \tilde{\Delta}\right)\right\} .
\end{gathered}
$$

The solution is sought in a Hilbert space defined by the scalar product

$$
(a, b)=\int d \mathbf{c} \chi_{0}(\mathbf{c}) a^{*}(\mathbf{c}) b(\mathbf{c})
$$

The relevant eigenvalue problem is

$$
(-i \mathbf{k} \cdot \mathbf{c}+\mathcal{L}) \phi_{i}=\omega_{i}(k) \phi_{i}
$$

It is understood that the index $i$ may be discrete or continuous. The hydrodynamic spectrum can be defined precisely as follows. The linearized Boltzmann equation provides exact 
balance equations for the moments of $\Delta$ corresponding to the density, flow velocity, and temperature. The latter are the $d+2$ hydrodynamic fields. The spectrum of these balance equations can be calculated without approximation in the limit $\mathbf{k}=0$. For elastic collisions, there is a $(d+2)$-fold degenerate point at zero eigenvalue, corresponding to the conservation laws for binary collisions. Their perturbation at finite $\mathbf{k}$ defines the hydrodynamic modes more generally. For inelastic collisions, the spectrum is again $d+2$ points, now at $0, \zeta_{0} / 2,-\zeta_{0} / 2$, with the latter being $d$-fold degenerate. Again, the hydrodynamic modes more generally are defined as those solutions to (7) that are continuously connected as functions of $k$ to these special $k=0$ solutions. For elastic collisions, it is possible to prove analyticity about $k=0$ [8], and it is assumed this is the case here as well.

The first step is to show that $\mathcal{L}$ includes the spectrum of the balance equations at $k=0$, and to determine the corresponding eigenfunctions. For elastic collisions, the stationary state is given by $J\left[f_{M}, f_{M}\right]=0$, whose solution is the Maxwellian. Differentiating this stationary equation with respect to the hydrodynamic fields leads to the linearized Boltzmann operator acting on combinations of $1, \mathbf{c}, c^{2}$ independently being zero. These are the $d+2$ eigenfunctions with zero eigenvalue. A similar analysis works for the inelastic case, using the stationary condition (3). Straightforward calculations give

$$
\begin{gathered}
\mathcal{L} \phi_{i}^{(0)}=\omega_{i}^{(0)} \phi_{i}^{(0)}, \quad i=1, . ., d+2, \\
\phi^{(0)} \rightarrow\left\{d+1+\mathbf{c} \cdot \frac{\partial \ln \chi_{0}}{\partial \mathbf{c}},-d-\mathbf{c} \cdot \frac{\partial \ln \chi_{0}}{\partial \mathbf{c}},-\frac{\partial \ln \chi_{0}}{\partial \mathbf{c}}\right\}, \\
\omega_{i}^{(0)} \rightarrow\{0, \zeta / 2,-\zeta / 2\} .
\end{gathered}
$$

The evaluation of the hydrodynamic spectrum at finite $k$ is now a well-defined technical problem. In general, the eigenvalues depend on both $k$ and the restitution coefficient $\alpha$. It is known from the Navier-Stokes equations that the behavior for small $k(\alpha)$ is not uniform with respect to $\alpha(k)$, so that a perturbation expansion in one or the other does not provide the entire mode structure. Here, for illustration, only the results for small $k$ are reported. The expansion is defined by $\omega_{i}(k)=\omega_{i}^{(0)}+i k \omega_{i}^{(1)}+k^{2} \omega_{i}^{(2)}+\cdots$, and $\phi_{i}(k)=\phi_{i}^{(0)}+i k \phi_{i}^{(1)}+k^{2} \phi_{i}^{(2)}+\cdots$. The leading terms are those of Eqs. (9) and (10). Since $\mathcal{L}$ is not self-adjoint, it is necessary to introduce a set of functions $\left\{\psi_{i}\right\}$ that are biorthogonal to $\left\{\phi_{i}\right\}$. The leading terms of a similar expansion in $k$ are found to be

$$
\psi_{i}^{(0)} \rightarrow\left\{1, \frac{c^{2}}{d}+\frac{1}{2}, \widehat{\mathbf{k}} \cdot \mathbf{c}, \widehat{\mathbf{e}}^{(i)} \cdot \mathbf{c}\right\},
$$


where $\left\{\widehat{\mathbf{k}}, \widehat{\mathbf{e}}^{(i)}\right\}$ are $d$ pairwise orthogonal unit vectors. The results to second order in $k$ are $\omega_{i}^{(1)}=0$,

$$
\begin{gathered}
\omega_{i}^{(2)}=\left(\psi_{i}^{(0)}, \widehat{\mathbf{k}} \cdot \mathbf{c} \phi_{i}^{(1)}\right)+\delta_{i 2} \zeta_{0}\left[\chi_{0}, \chi_{0} \mathcal{Q}_{1} \phi_{2}^{(2)}\right] \\
\phi_{i}^{(1)}=\mathcal{Q}_{i}\left(\mathcal{L}-\omega_{i}^{(0)}\right)^{-1} \widehat{\mathbf{k}} \cdot \mathbf{c} \phi_{i}^{(0)} \\
\phi_{i}^{(2)}=-\mathcal{Q}_{i}\left(\mathcal{L}-\omega_{i}^{(0)}\right)^{-1} \widehat{\mathbf{k}} \cdot \mathbf{c} \phi_{i}^{(1)}
\end{gathered}
$$

The operators $\mathcal{Q}_{i}=1-\mathcal{P}_{i}$ are projections orthogonal to $\phi_{i}^{(0)}$ with $\mathcal{P}_{i} a=\phi_{i}^{(0)}\left(\psi_{i}^{(0)}, a\right)$. The constants $\omega_{i}^{(2)}$ are transport coefficients which are identified from a similar calculation using the Navier-Stokes equations. For example, the shear viscosity $\eta$ is

$$
\eta=\frac{m v_{0} \omega_{4}^{(2)}}{\sigma^{d-1}}=-\frac{m v_{0}}{\sigma^{d-1}}\left(c_{x} c_{y},\left(\mathcal{L}+\frac{1}{2} \zeta_{0}\right)^{-1} c_{x} \frac{\partial \ln \chi_{0}}{\partial c_{y}}\right) .
$$

This agrees with the result obtained from the Chapman-Enskog method [13]. A more complete connection with the latter can be established as follows. Assuming that the hydrodynamic spectrum dominates for long times and small $k$, the solution to the Boltzmann equation for small perturbations becomes

$$
f \rightarrow f_{h c s}(\mathbf{v})\left[1+\sum_{i=1}^{d+2} \phi_{i}(k) \delta y_{i}^{h}(\mathbf{k}, s)\right] .
$$

Here $\delta y_{i}^{h}(\mathbf{k}, s)$ are the hydrodynamic fields

$$
\delta y_{i}^{h}(\mathbf{k}, s)=\left(\psi_{i}, \tilde{\Delta}(\mathbf{k}, s)\right)=e^{s \omega_{i}(k)}\left(\psi_{i}, \tilde{\Delta}(\mathbf{k}, 0)\right)
$$

The connection between these fields and the macroscopic density, temperature, and flow velocity is obtained for small $k$ by using $\psi_{i} \rightarrow \psi_{i}^{(0)}$ in (17). The fields $\delta y_{i}^{h}(\mathbf{k}, s)$ represent components of the initial distribution that obey the hydrodynamic equations for all times, whereas the density, temperature, and flow velocity become equal to these only on the long space and time scales [8]. Use of $\phi_{i}(k) \rightarrow \phi_{i}^{(0)}+i k \phi_{i}^{(1)}$ for small $k$ in (16) leads to a result that is in agreement with the Navier-Stokes approximation of the Chapman-Enskog method [14].

The existence of a hydrodynamic spectrum is relevant only if the corresponding modes dominate for long times and long wavelengths. A sufficient condition is that the magnitude of the hydrodynamic eigenvalues be smaller than that of all other parts of the spectrum. This is the case for elastic collisions, but the proof does not extend directly to $-i \mathbf{k} \cdot \mathbf{c}+\mathcal{L}$ here and the question remains open in general [15]. In fact, the mode at $\zeta_{0} / 2$ gives reason for concern since 
it might become comparable to the microscopic excitations at large inelasticity. To explore this point, it is instructive to consider a model for the Boltzmann equation that preserves the essential physics of a non-trivial HCS, exact balance equations, and hydrodynamic modes, as described above. The Boltzmann collision operator can be written

$$
J[f, f]=-\nu(f-g),
$$

where $\nu f$ is the "loss" contribution and $\nu g$ is the "gain" contribution. Both the collision frequency $\nu$ and $g$ are specified positive functionals of $f$.

The class of models to be considered here are characterized by the following two simplifications: 1) $\nu$ is independent of $\mathbf{v}$ and, 2) $\nu$ and $g$ are functionals of $f$ only through its moments with respect to $1, \mathbf{v}, v^{2}$. The first condition is similar to that of "Maxwell models" that have been introduced recently [10]. The second condition means that $\nu$ and $g$ depend on the state of the system only through the hydrodynamic fields. The forms of these functionals are subjected to preserve the exact properties of the collision operator required by the balance equations,

$$
\int d \mathbf{v}\left(\begin{array}{c}
1 \\
\mathbf{v} \\
m v^{2}
\end{array}\right) g(\mathbf{r}, \mathbf{v}, t)=\left(\begin{array}{c}
n \\
n \mathbf{u} \\
n T d\left(\lambda^{2}+\frac{m u^{2}}{T d}\right)
\end{array}\right) .
$$

Here $\mathbf{u}$ is the flow velocity and $\lambda^{2} \equiv 1-\zeta / \nu$. In principle, $\zeta$ is the same functional of $f$ as obtained from the Boltzmann equation, although it can be eventually approximated similarly to $\nu$ and $g$. I any case, Eq. (21) must be preserved. In the following, we will assume that both $\zeta$ and $\nu$ scale as $T^{1 / 2}$ in order to mimic hard sphere behavior. With the above class of models, it is obtained from Eq. (3)

$$
\chi_{0}(\mathbf{c})=\int_{1}^{\infty} d x P(x)(\lambda x)^{-d} g^{*}(\mathbf{c} / \lambda x)
$$

where $P(x) \equiv p x^{-(1+p)}$ with $p=2 \nu / \zeta$. The dimensionless gain function $g\left(\mathbf{v} ; y^{h}(\mathbf{r}, t)\right)=$ $n_{H}\left(\lambda v_{0}\right)^{3} g^{*}(\mathbf{c} / \lambda)$ has been introduced. Further investigation requires specification of $g^{*}(\mathbf{c} / \lambda)$. However, it is easily verified that moments of degree equal or greater than $p$ are not finite if the corresponding moments of $g^{*}$ exist. This is consistent with a similar result for the Maxwell models where algebraic decay of $\chi_{0}(\mathbf{c})$ is observed for large velocities, also due to a slower decay of the loss term than the gain term. 
Linearization of the kinetic model around this HCS leads again to Eq. (44), but with a simpler form for $\mathcal{L}$. It has a decomposition into the subspace of $\phi_{i}^{(0)}$ and its complement, $\mathcal{L}=\mathcal{P} \mathcal{L} \mathcal{P}+\mathcal{Q} \mathcal{L} \mathcal{Q}$, where $\mathcal{P}=\sum \mathcal{P}_{i}$ and $\mathcal{Q}=1-\mathcal{P}$. In the subspace of $\phi_{i}^{(0)}$ the spectrum is just the $d+2$ points of (10),

$$
\mathcal{P} \mathcal{L} \mathcal{P}=\sum_{i=1}^{d+2} \omega_{i}^{(0)} \mathcal{P}_{i}
$$

In the complementary subspace the operator is

$$
\mathcal{Q} \mathcal{L} \mathcal{Q}=\nu \mathcal{Q}+\chi_{0}^{-1} \frac{\zeta_{0}}{2} \frac{\partial}{\partial \mathbf{c}} \cdot\left(\mathbf{c} \chi_{0} \mathcal{Q}\right)
$$

Interestingly, there is no explicit dependence on the gain function $g^{*}$ except through $\chi_{0}$. For elastic collisions, the second term is missing and the model becomes the linearized BhatnagerGross-Krook model [16], with a single degenerate point in the spectrum representing all the microscopic excitations of the Boltzmann operator. It is somewhat easier to analyze the corresponding adjoint problem

$$
\nu \phi-\frac{1}{2} \zeta_{0} \mathbf{c} \cdot \frac{\partial}{\partial \mathbf{c}} \phi=\omega \phi, \quad \phi=\mathcal{Q}^{\dagger} \phi
$$

where $\mathcal{Q}^{\dagger}$ is the adjoint of $\mathcal{Q}$. Solutions to this equation exist from the class of homogeneous functions

$$
\phi \propto \prod_{i=1}^{d} c_{i}^{q_{i}}, \quad \sum_{i=1}^{d} q_{1}=q .
$$

The property $\mathcal{Q}^{\dagger} \phi=\phi$ can be satisfied with a suitable choice of the linear combinations. The corresponding eigenvalue is $\omega=\nu-q \zeta_{0} / 2$. Thus the spectrum of $\mathcal{L}$ includes a continuum with $\Re \omega \leq \nu$. Of critical interest for hydrodynamics is whether the lower bound for this continuum on the real axis intersects the hydrodynamic point spectrum. The bound is determined by the maximum degree of such homogeneous eigenfunctions admitted in the Hilbert space. The condition is that $\left\|c^{q}\right\|^{2}=\left(c^{q}, c^{q}\right)$ exists. Using Eq. (20), this becomes

$$
\begin{gathered}
\left\|c^{q}\right\|^{2}=\lambda^{2 \Re q} p M_{q} \int_{1}^{\infty} d x x^{2 \Re q-(1+p)}, \\
M_{q}=\int_{0}^{\infty} d \mathbf{c} c^{2 \Re q} g^{*}(c) .
\end{gathered}
$$

For finite $M_{q}$, the value of $q$ is bounded from above by $\Re q<q_{m}=p / 2=\nu / \zeta_{0}$. The continuous spectrum therefore is restricted by $\nu / 2<\Re \omega \leq \nu$. This means that the discrete spectrum is isolated from the continuum for all $\zeta_{0} / \nu<1$. But $\zeta_{0} / \nu$ must be smaller than 
unity because of the third condition required by Eq. (19). This condition is related with the fact that only a fraction of the energy of the particles can be lost in collisions. The continuum is singular in the sense that it is independent of the restitution coefficient but it is present only for finite inelasticity.

For this class of models, it is seen that the hydrodynamic spectrum at $k=0$ is isolated from the rest of the spectrum. The model also allows exact calculation of the hydrodynamic eigenvalues $\omega_{i}(k)$ at finite $k$, without the restrictions of the perturbation theory calculation above. For elastic collisions, such a calculation shows that the hydrodynamic modes exist only for $k$ less than some maximum value. It is expected that a similar condition applies in the granular case as well.

The research of J.W.D. was supported by Department of Energy Grants DE-FG0398DP00218 and DE-FG02ER54677. The research of J.J.B. was partially supported by the Ministerio de Ciencia y Tecnología (Spain) through Grant No. BFM2002-00307.

[1] T. P. C van Noije and M. H. Ernst, in Granular Gases, T. Pöschel and S. Luding, eds. (Lecture Notes in Physics 564, Springer, Berlin, 2001); J. W. Dufty, Adv. Complex Sys. 4, 397 (2001).

[2] J. T. Jenkins and M. W. Richman, Arch. Ration. Mech. Anal. 87, 355 (1985).

[3] C. K. W. Lun, S. B. Savage, D. J. Jeffrey, and N. Chepurnuy, J. Fluid Mech. 140, 223 (1984).

[4] A. Goldshtein and M. Shapiro, J. Fluid Mech. 282, 41, 1995.

[5] N. Sela and I. Goldhirsch, J. Fluid Mech. 361, 41 (1998).

[6] J. J. Brey, J. W. Dufty, C. S. Kim, and A. Santos, Phys. Rev. E 58, 4638 (1998).

[7] J. J. Brey and D. Cubero, in Granular Gases, T. Pöschel and S. Luding,(Lecture Notes in Physics 564, Springer, Berlin, 2001).

[8] J. A. McLennan, Introduction to Nonequilibrium Statistical Mechanics, (Prentice-Hall, New Jersey, 1989).

[9] J. J. Brey, F. Moreno, and J. W. Dufty, Phys. Rev. E 54, 445 (1996).

[10] M. H. Ernst and R. Brito, Euro. Phys. Lett. 58, 182 (2002).

[11] T. P. C van Noije and M. H. Ernst, in Gran. Matt. 1, 57 (1998).

[12] J. J. Brey, M. J. Ruiz-Montero, and D. Cubero, Phys. Rev. E 54, 3664 (1998).

[13] J. W. Dufty and J. J. Brey, J. Stat. Phys. 109, 433 (2002). 
[14] J. J. Brey, J. W. Dufty, and M. J. Ruiz-Montero, e-print cond-mat/0302180.

[15] I. Goldhirsh, in Granular Gases, T. Pöschel and S. Luding, eds. (volume 564 of Lecture Notes in Physics 564, Springer, Berlin, 2001).

[16] C. Cercignani, Theory and Application of the Boltzmann Equation, (Elsevier, NY, 1975). 